\title{
Thermodynamics with pressure and volume under charged particle absorption
}

\author{
Bogeun Gwak \\ Department of Physics and Astronomy, Sejong University, \\ Seoul 05006, Republic of Korea \\ E-mail: rasenis@sejong.ac.kr
}

ABSTRACT: We investigate the variation of the charged anti-de Sitter black hole under charged particle absorption by considering thermodynamic volume. When the energy of the particle is considered to contribute to the internal energy of the black hole, the variation exactly corresponds to the prediction of the first law of thermodynamics. Nevertheless, we find the decrease of the Bekenstein-Hawking entropy for extremal and near-extremal black holes under the absorption, which is an irreversible process. This violation of the second law of thermodynamics is only found when considering thermodynamic volume. We test the weak cosmic censorship conjecture affected by the violation. Fortunately, the conjecture is still valid, but extremal and near-extremal black holes do not change their configurations when any particle enters the black hole. This result is quite different from the case in which thermodynamic volume is not considered.

Keywords: Black Holes, Classical Theories of Gravity, Models of Quantum Gravity

ArXIV EPRINT: 1709.08665 


\section{Contents}

1 Introduction 1

2 Thermodynamic volume in charged AdS black hole 3

3 Thermodynamics with pressure and volume under charged particle absorption

4 Weak cosmic censorship conjecture with pressure and volume

5 Summary

\section{Introduction}

Black holes have an event horizon through which no particle can escape from its gravity, even if the particles are photons. Classically, no energy or matter can reach an observer located outside a black hole. However, in consideration of a quantum effect, a small portion of energy can be radiated to the outside of the horizon in a black hole spacetime. The temperature of the black hole is defined from this radiated energy, and the black hole can be treated as a thermal system having the Hawking temperature [1, 2]. Further, black holes have an irreducible mass, which is a property that increases for an irreversible process [36]. The irreducible mass is similar to the entropy in a thermal system, and based on this similarity, the entropy of a black hole is obtained from the irreducible mass. This entropy is the Bekenstein-Hawking entropy of a black hole [7, 8] and is proportional to the area of the horizon. Using these two thermal properties, temperature and entropy, the laws of thermodynamics are constructed for the black hole as a thermal system.

An interesting conjecture called the weak cosmic censorship conjecture has been applied to the horizon of a black hole, in which the horizon of the black hole should cover its inside because the singularity of the black hole geometry is located at the center of the black hole spacetime $[9,10]$. This conjecture is inevitable to save the causality of the spacetime from a naked singularity. Although the conjecture is generally suggested for black holes, its validity should be tested for each case because there is no general procedure to prove it. Further, the validity of the conjecture depends on the methods of investigation. For the Kerr black hole, the conjecture is valid under the addition of a particle [11]. However, the near-extremal Kerr black hole can be overspun beyond its extremal bound by a particle, making the conjecture invalid [12]. To resolve this invalidity, the conjecture has been proven to be valid for the Kerr black hole in consideration of the self-force effect [13-15]. The validity of this conjecture has been tested for the Reissner-Nordström (RN) black hole with the back-reaction effect $[16,17]$. In addition, various investigations have been 
conducted on the conjecture for not only black holes of Einstein's theory of gravity, but also anti-de Sitter (AdS), lower-dimensional, and higher-dimensional black holes [18-30]. From a thermodynamic point of view, the conjecture is quite consistently related to the laws of thermodynamics. If the entropy of the black hole increases, as ensured by the second law for an irreversible process, the horizon can cover the inside of a black hole, as the conjecture suggests. In addition, in the process, the variation of a black hole is consistent with the first law of thermodynamics under particle absorption [31, 32].

Thermal properties play an important role in AdS spacetime. The gravity theory in $D$-dimensional AdS spacetime is associated with the conformal field theory (CFT) defined on the boundary of the AdS spacetime. This association is called the AdS/CFT correspondence [33-36]. Under this duality, the thermal properties of the AdS black hole are also found in dual CFT so that CFT is given at a finite temperature [37]. Currently, there are various applications of AdS/CFT duality. One of the representative applications is the anti-de Sitter/quantum chromodynamics (AdS/QCD) correspondence [38-41]. Another is the anti-de Sitter/condensed matter theory (AdS/CMT) correspondence [42, 43]. Because each solution of black holes is based on various gravity theories, its dual theory and physical interpretation depend on the black hole considered. Further, the instability of black holes in perturbation or thermodynamics affects the states of dual CFT. For example, the charged AdS black hole is mainly related to AdS/CMT applications. In the $(2+1)$ dimensional charged AdS black hole, its dual theory is associated with the holographic superconductor [44-46]. In addition, the Fermi-Luttinger liquid is a model resembling the dual theory $[47,48]$.

The cosmological constant is a parameter that plays an important role in determining the asymptotic topology of a black hole spacetime. In the action of Einstein's theory of gravity, the cosmological constant is fixed. Therefore, it is set to a constant value at any time. Recently, various interesting studies have been conducted on the thermodynamics of black holes wherein the cosmological constant was set as a dynamic variable and interpreted as a pressure. In fact, the cosmological constant was first considered as a dynamic variable a long time ago $[49,50]$. Furthermore, the pressure of the black hole spacetime is associated with the cosmological constant [51, 52], and its thermal conjugate is found to be a thermodynamic volume $[53,54]$. Under the cosmological constant as a dynamic variable, the mass of the black hole corresponds to the enthalpy of the black hole system [55]. Owing to the pressure-volume contribution, the first law of thermodynamics is extended to have a $P V$ term [56]. This considerably affects the thermal phase of the black hole, and various phenomena have already been investigated, such as Van der Waals fluids, re-entrant phase transitions, and holographic heat engines [57-63]. A study on the growth of the horizon area in the Schwarzschild-de Sitter black hole was recently reported [64].

In this paper, we prove that the variation in the $D$-dimensional charged AdS black hole including four dimensions due to charged particle absorption follows the first law of thermodynamics in consideration the thermodynamic volume term. Under the variation, the energy of the particle is considered to contribute to the internal energy of the black hole, rather than its enthalpy. Further, we investigate the second law of thermodynamics for this black hole. Because particle absorption is an irreversible process, the entropy of 
the black hole should increase. It has been already proved that the satisfaction of the first law of thermodynamics is a necessary condition to ensure satisfaction of the second law of thermodynamics under particle absorption $[31,32]$ in the absence of a thermodynamic volume term. Nevertheless, if the second law of thermodynamics is not valid under the absorption, it would be the first case where the second law of thermodynamics is violated when the first law of thermodynamics is satisfied, which would only be observed when considering the pressure and volume term. The second law of thermodynamics plays an important role in physical processes such as the collision of black holes [65]. Considering the thermodynamic volume term, we assume the cosmological constant as a dynamic variable in the metric of the black hole. Under this assumption, we test the weak cosmic censorship conjecture under charged particle absorption. Moreover, in the Einstein-Maxwell action, the cosmological constant is not a dynamic variable; therefore, we cannot test the conjecture at the level of equations of motion. Thus, our analysis in terms of the particle absorption is probably the only method to investigate the conjecture from the variation in the black hole including the pressure and volume term.

This paper is organized as follows. In section 2, the charged AdS black hole is introduced, and the laws of thermodynamics are presented along with the dynamic cosmological constant. In section 3, we establish the first law of thermodynamics under charged particle absorption. Further, the second law of thermodynamics is shown to be violated in specific cases. In section 4, we describe the investigation of the weak cosmic censorship conjecture for extremal and near-extremal black holes. In section 5 , we briefly summarize our results.

\section{Thermodynamic volume in charged AdS black hole}

The Einstein-Maxwell action with the cosmological constant in the $D$-dimensional spacetime is given as

$$
S=-\frac{1}{16 \pi} \int d^{D} x \sqrt{-g}\left(R-F_{\mu \nu} F^{\mu \nu}-2 \Lambda\right),
$$

where the spacetime dimensions are denoted as $D \geq 4$. The Maxwell field strength $F_{\mu \nu}$ and electric potential $A_{\mu}$ are

$$
F_{\mu \nu}=\partial_{\mu} A_{\nu}-\partial_{\nu} A_{\mu}, \quad A=-\frac{Q}{r^{D-3}} d t
$$

The equations of motion in eq. (2.1) have a static solution for the charged AdS black hole. The metric of the black hole is in $D$-dimensional spacetime

$$
d s^{2}=-f(r) d t^{2}+f(r)^{-1} d r^{2}+r^{2} d \Omega_{D-2}, \quad f(r)=1-\frac{2 M}{r^{D-3}}+\frac{Q^{2}}{r^{2 D-6}}+\frac{r^{2}}{\ell^{2}},
$$

where the $D-2$-dimensional sphere is analytically expressed as

$$
d \Omega_{D-2}=\sum_{i=1}^{D-2}\left(\prod_{j=1}^{i} \sin ^{2} \theta_{j-1}\right) d \theta_{i}^{2}, \quad \theta_{0} \equiv \frac{\pi}{2}, \quad \theta_{D-2} \equiv \phi .
$$


The metric components are determined in terms of mass and charge parameters $M$ and $Q$, respectively, with the AdS radius $\ell$ in eq. (2.3). These parameters are proportional to the mass $M_{\mathrm{b}}$, electric charge $Q_{\mathrm{b}}$, and cosmological constant $\Lambda$ [66].

$$
M_{\mathrm{b}}=\frac{(D-2) \Omega_{D-2}}{8 \pi} M, \quad Q_{\mathrm{b}}=\frac{(D-2) \Omega_{D-2}}{8 \pi} Q, \quad \Lambda=-\frac{(D-1)(D-2)}{2 \ell^{2}}, \quad \Omega_{D-2}=\frac{2 \pi^{\frac{D-1}{2}}}{\Gamma\left(\frac{D-1}{2}\right)},
$$

where we set $G=1$ and $\hbar=1$ in all dimensions for simplicity. The thermal properties can be defined on the horizon $r_{\mathrm{h}}$ of the black hole. The Hawking temperature, BekensteinHawking entropy, and electric potential are given as

$$
T_{\mathrm{h}}=\frac{1}{2 \pi \ell^{2}}\left(r_{\mathrm{h}}-\frac{(D-3) Q^{2} \ell^{2}}{r_{\mathrm{h}}^{2 D-5}}+\frac{(D-3) M \ell^{2}}{r_{\mathrm{h}}^{D-2}}\right), \quad S_{\mathrm{h}}=\frac{A_{\mathrm{h}}}{4}=\frac{\Omega_{D-2} r_{\mathrm{h}}^{D-2}}{4}, \quad \Phi_{\mathrm{h}}=\frac{Q}{r_{\mathrm{h}}^{D-3}} .
$$

Then, the thermodynamic laws can be constructed for the black hole. Recently, an interesting approach is followed to treat the cosmological constant as a thermodynamic variable. From this point of view, the cosmological constant is not a fixed value. Its actual value can be obtained from the vacuum expectation value of the theory considered, and it can vary under a perturbation [54]. Although the variation of the cosmological constant is not concrete in the Lagrangian theory, the cosmological constant as a thermodynamic variable shows quite consistent behaviors with other thermodynamic variables $[53,56]$. In this extended thermodynamics, the cosmological constant plays the role of pressure $P$, and its conjugate variable is the thermodynamic volume of the black hole $V_{\mathrm{b}}$. The definitions of thermodynamic pressure and volume are in $D$-dimensional AdS spacetime [68]

$$
P=-\frac{\Lambda}{8 \pi}=\frac{(D-1)(D-2)}{16 \pi \ell^{2}}, \quad V_{\mathrm{b}}=\frac{\Omega_{D-2}}{D-1} r_{\mathrm{h}}^{D-1} .
$$

When we consider the pressure term in the laws of thermodynamics, the key difference is that the mass is now enthalpy in the first law of thermodynamics $[54,55]$. Thus, the first law of thermodynamics determines the infinitesimal change of the mass of the black hole as $[67,68]$

$$
d M_{\mathrm{b}}=T_{\mathrm{h}} d S_{\mathrm{h}}+\Phi_{\mathrm{h}} d Q+V_{\mathrm{h}} d P
$$

where $M_{\mathrm{b}}$ plays the role of enthalpy. The enthalpy is related to the internal energy of the black hole $U_{\mathrm{b}}$ and the $P V_{\mathrm{b}}$ term as

$$
M_{\mathrm{b}}=U_{\mathrm{b}}+P V_{\mathrm{b}}
$$

Therefore, the variation of the mass causes the rebalancing of not only the horizon and electric charge, but also the AdS radius in the $P V_{\mathrm{b}}$ term. In the following section, we investigate the change in the black hole under charged particle absorption when the AdS radius is assumed to be infinitesimally varied because of the absorption. 


\section{Thermodynamics with pressure and volume under charged particle absorption}

We assume that the charged AdS black hole is varied when it absorbs a charged particle. When the black hole absorbs the particle, the conserved quantities of the particle can perturb both the mass and charge of the black hole, and the AdS radius is affected by these changes owing to the contribution of thermodynamic pressure and volume. To analyze this charged particle absorption, we obtain the relation between the conserved quantities of the particle because the conserved quantities of the black hole are changed as much as those of the particle. Then, the Hamiltonian of the charged particle under an electric potential $A_{\mu}$ is given as

$$
\mathcal{H}=\frac{1}{2} g^{\mu \nu}\left(p_{\mu}-q A_{\mu}\right)\left(p_{\nu}-q A_{\nu}\right),
$$

and the Hamiltonian equations are separable under the Hamilton-Jacobi action [69]. The momentum $p_{\mu}$ is obtained in terms of a partial derivative of the Hamilton-Jacobi action

$$
S=\frac{1}{2} m^{2} \lambda-E t+L \phi+S_{r}(r)+\sum_{i=1}^{D-3} S_{\theta_{i}}\left(\theta_{i}\right), \quad p_{\mu}=\partial_{\mu} S .
$$

The Hamilton-Jacobi action describes a particle having a mass $m^{2}$, and the affine parameter is $\lambda$. The conserved quantities $E$ and $L$ are assumed from the translation symmetries of the metric in eq. (2.3). Owing to the $D$-dimensional solution, the black hole includes a $D-2$-dimensional sphere $\Omega_{D-2}$. The angular momentum $L$ is defined as the conserved quantity from the translation symmetry of the last angle coordinate of $\Omega_{D-2}$. Thus, the summation in eq. (3.2) runs from $i=1$ to $D-3$. To solve the Hamilton-Jacobi equations, we express the inverse metric including a $D-2$-dimensional sphere

$$
g^{\mu \nu} \partial_{\mu} \partial_{\nu}=-f(r)^{-1}\left(\partial_{t}\right)^{2}+f(r)\left(\partial_{r}\right)^{2}+r^{-2} \sum_{i=1}^{D-2}\left(\prod_{j=1}^{i} \sin ^{-2} \theta_{j-1}\right)\left(\partial_{\theta_{i}}\right)^{2} .
$$

The Hamilton-Jacobi equation is

$$
\begin{aligned}
-2 \frac{\partial S}{\partial \lambda}=-m^{2}= & -f(r)^{-1}\left(-E-q A_{t}\right)^{2}+f(r)\left(\partial_{r} S_{r}(r)\right)^{2} \\
& +r^{-2} \sum_{i=1}^{D-3}\left(\prod_{j=1}^{i} \sin ^{-2} \theta_{j-1}\right)\left(\partial_{\theta_{i}} S_{\theta_{i}}\left(\theta_{i}\right)\right)^{2}+r^{-2}\left(\prod_{j=1}^{D-2} \sin ^{-2} \theta_{j-1}\right)(L)^{2}
\end{aligned}
$$

which is divided by separate variables, $\mathcal{K}$ and $R_{i}$.

$\mathcal{K}=-m^{2} r^{2}+\frac{r^{2}}{f(r)}\left(-E+\frac{Q q}{r^{D-3}}\right)^{2}-r^{2} f(r)\left(\partial_{r} S_{r}(r)\right)^{2}, \quad R_{i}^{2}=\left(\partial_{i} S_{\theta_{i}}\left(\theta_{i}\right)\right)^{2}+\sin ^{2} \theta_{i} R_{i+1}^{2}$,

where two variables are defined as

$$
\mathcal{K}=R_{1}^{2}, \quad L=R_{D-2} .
$$


Then, we can determine entire equations of motion. The radial- and $\theta$-directional equations are sufficient to obtain the relation between the energy and electric charge of the particle. The momenta of the particle are

$$
p^{r} \equiv \frac{\partial r}{\partial \lambda}=f(r) \sqrt{-\frac{\mathcal{K}+m^{2} r^{2}}{r^{2} f(r)}+\frac{1}{f(r)^{2}}\left(E-\frac{Q q}{r^{D-3}}\right)^{2}}, \quad p^{\theta} \equiv \frac{\partial \theta}{\partial \lambda}=\frac{1}{r^{2}} \sqrt{\mathcal{K}-\sin ^{2} \theta_{1} R_{2}^{2}} .
$$

We attempt to determine the variation in the black hole when it absorbs a charged particle. The particle is supposed to be absorbed by the black hole when it passes through the outer horizon $r_{\mathrm{h}}$ because the conserved quantities of the particle are not distinguishable anymore from those of the black hole at that moment by an observer outside of the horizon. By removing the separate variable $\mathcal{K}$ in eq. (3.7), we obtain the relation between the conserved quantities and momenta for a given radial location $r$. Then, at the outer horizon $r_{h}$, the conserved quantities of the particle are assimilated into those of the black hole. At the limit of the outer horizon, the energy relation between conserved quantities and momenta is obtained as

$$
E=\frac{Q}{r_{\mathrm{h}}^{D-3}} q+\left|p^{r}\right|
$$

in which various dependencies between variables are reduced to this simple relation. A positive sign is required in front of the $\left|p^{r}\right|$ term. The particle enters the black hole in the positive flow of time. At this moment, the energy of the particle should be defined as a positive value so that the signs of $E$ and $\left|p^{r}\right|$ are both positive [3, 4]. Note that we consider the energy dependent on the electric potential term. However, the potential is independent of the flow of time and only related to the interaction between the particle and black hole. Thus, the total value of energy under the sum of the potential is not important, and we simply choose the positive sign for $\left|p^{r}\right|$.

By absorbing the charged particle, the black hole is varied by as much as the variation in the particle, assuming no loss of conserved quantities in this process. This is supported by the change in the black hole following the first law of thermodynamics. The charge in the particle $q$ is coincident to the change of the charge of the black hole $d Q_{\mathrm{b}}$. Because the energy of the particle is only given as $q$ and $\left|p^{r}\right|$ at the horizon in eq. (3.8), we must find a thermodynamic potential of which the variables also change by $q$ and $\left|p^{r}\right|$. If we assume that the energy of the particle changes the internal energy of the black hole, the internal energy is expressed as $U_{\mathrm{b}}\left(Q_{\mathrm{b}}, S_{\mathrm{b}}, V_{\mathrm{b}}\right)$, and its variation is given as $d Q_{\mathrm{b}}, d S_{\mathrm{b}}$, and $d V_{\mathrm{b}}$. Fortunately, these variables will be expressed in terms of those of the particle. The energy and electric charge of the particle are

$$
E=d U_{\mathrm{b}}=d\left(M_{b}-P V_{\mathrm{b}}\right), \quad q=d Q_{\mathrm{b}} .
$$

Then, the energy relation in eq. (3.8) is rewritten as

$$
d U_{\mathrm{b}}=\frac{Q}{r_{\mathrm{h}}^{D-3}} d Q_{b}+\left|p^{r}\right| .
$$

Thus, the charged particle changes the black hole by as much as $\left(d U_{\mathrm{b}}, d Q_{\mathrm{b}}\right)$, and the change in the black hole volume induces a change in its conjugate variable, pressure. Under the 
charged particle absorption, the changed variables are $\left(d M_{\mathrm{b}}, d Q_{\mathrm{b}}, d \ell\right)$. The other variables depend on these. To rewrite eq. (3.10) to the first law of thermodynamics, we need to find $d S_{\mathrm{h}}$ changed by the absorption. Under the variation,

$$
d S_{\mathrm{h}}=\frac{1}{4}(D-2) \Omega_{D-2} r_{\mathrm{h}}^{D-3} d r_{\mathrm{h}},
$$

where the change of the outer horizon $d r_{\mathrm{h}}$ should be rewritten as independent variables such as $\left(q,\left|p^{r}\right|\right)$ of the particle. The particle absorption varies the function $f(r)$, and its change is the reason for the moved outer horizon $r_{\mathrm{h}}+d r_{\mathrm{h}}$. The infinitesimally small movement of the outer horizon $d r_{\mathrm{h}}$ satisfies

$$
d f_{\mathrm{h}}=\frac{\partial f_{\mathrm{h}}}{\partial M_{\mathrm{b}}} d M_{\mathrm{b}}+\frac{\partial f_{\mathrm{h}}}{\partial Q_{\mathrm{b}}} d Q_{\mathrm{b}}+\frac{\partial f_{\mathrm{h}}}{\partial \ell} d \ell+\frac{\partial f_{\mathrm{h}}}{\partial r_{\mathrm{h}}} d r_{\mathrm{h}}=0, \quad f_{\mathrm{h}}=f\left(M_{\mathrm{b}}, Q_{\mathrm{b}}, \ell, r_{\mathrm{h}}\right),
$$

with

$$
\begin{aligned}
\frac{\partial f_{\mathrm{h}}}{\partial M_{\mathrm{b}}} & =-\frac{16 \pi}{(D-2) \Omega_{D-2} r_{\mathrm{h}}^{D-3}}, & \frac{\partial f_{\mathrm{h}}}{\partial Q_{\mathrm{b}}} & =\frac{16 \pi Q}{(D-2) \Omega_{D-2} r_{\mathrm{h}}^{2 D-6}}, \\
\frac{\partial f_{\mathrm{h}}}{\partial \ell} & =-\frac{2 r_{\mathrm{h}}^{2}}{\ell^{3}}, & \frac{\partial f_{\mathrm{h}}}{\partial r_{\mathrm{h}}} & =-\frac{(2 D-6) Q^{2}}{r_{\mathrm{h}}^{2 D-5}}+\frac{2(D-3) M}{r_{\mathrm{h}}^{D-2}}+\frac{2 r_{\mathrm{h}}}{\ell^{2}} .
\end{aligned}
$$

In addition, the energy relation in eq. (3.10) is rewritten in terms of the enthalpy

$$
d M_{\mathrm{b}}-d\left(P V_{\mathrm{h}}\right)=\frac{8 \pi Q_{\mathrm{b}}}{(D-2) \Omega_{D-2} r^{D-3}} d Q_{\mathrm{b}}+\left|p^{r}\right| .
$$

By combining eqs. (3.12) and (3.14), we can remove the $d \ell$ term. Then, interestingly, except for the $\left|p^{r}\right|$ and $d r_{\mathrm{h}}$ variables, the others are removed. The change of the outer horizon becomes

$$
d r_{\mathrm{h}}=\frac{16 \pi r_{\mathrm{h}}^{4} \ell^{2}\left|p^{r}\right|}{\Omega_{D-2}(D-2)(D-3)\left(r_{\mathrm{h}}^{D+2}-2 M r_{\mathrm{h}}^{3} \ell^{2}+2 r_{\mathrm{h}}^{D} \ell^{2}\right)},
$$

which implies that the change of the outer horizon is proportional to the radial momentum. This originates from the balance between eqs. (3.12) and (3.14). Further, since $d P$ terms are all removed because of the balance, the change of the outer horizon in eq. (3.15) gives an identical result in an isobaric process. Again, the particle does not carry a corresponding charge to change the pressure term, leading to the identical result even if we consider variations to all dynamic variables. Therefore, under the energy relation, the variations of entropy and thermodynamic volume of the black hole are obtained as

$$
\begin{aligned}
d S_{\mathrm{h}} & =\frac{4 \pi r_{\mathrm{h}}^{D+1} \ell^{2}\left|p^{r}\right|}{(D-3)\left(r_{\mathrm{h}}^{D+2}-2 M r_{\mathrm{h}}^{3} \ell^{2}+2 r_{\mathrm{h}}^{D} \ell^{2}\right)}, \\
d V_{\mathrm{h}} & =\frac{16 \pi r_{\mathrm{h}}^{D+1} \ell^{2}\left|p^{r}\right|}{(D-2)(D-3)\left(r_{\mathrm{h}}^{D+2}-2 M r_{\mathrm{h}}^{3} \ell^{2}+2 r_{\mathrm{h}}^{D} \ell^{2}\right)} .
\end{aligned}
$$

Incorporating eqs. (2.6), (2.7), and (3.16), the energy relation of eq. (3.10) becomes the first law of thermodynamics

$$
d U_{\mathrm{h}}=\Phi_{\mathrm{h}} d Q_{b}+T_{\mathrm{h}} d S_{\mathrm{h}}-P d V_{\mathrm{h}}
$$




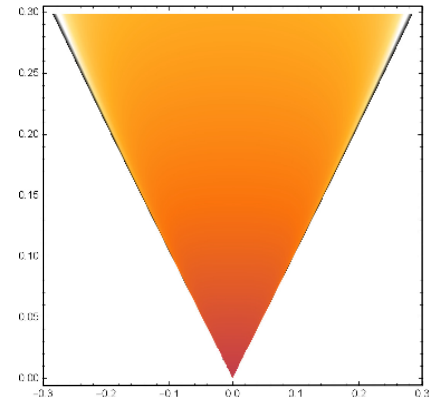

(a) $Q-M$ diagram for $D=4$.

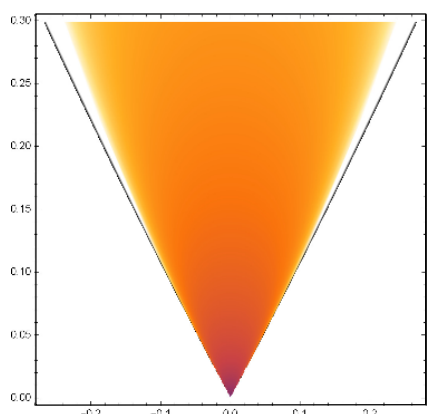

(b) $Q-M$ diagram for $D=5$.

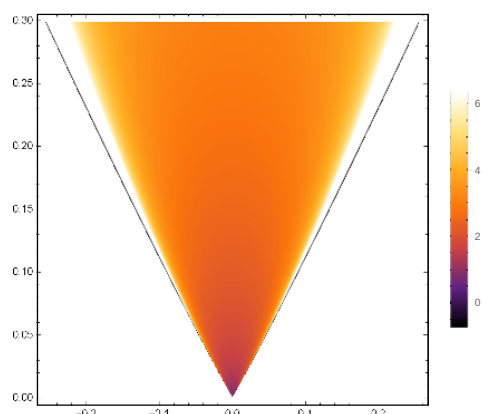

(c) $Q-M$ diagram for $D=6$.

Figure 1. $d S_{\mathrm{h}}$ in $Q-M$ diagrams with $\ell=1$.

Because the mass of the black hole is already defined as the enthalpy of the black hole, the internal energy can be exchanged with the enthalpy by the Legendre transformation. So,

$$
d M_{\mathrm{b}}=T_{\mathrm{h}} d S_{\mathrm{h}}+\Phi_{\mathrm{h}} d Q+V_{\mathrm{h}} d P
$$

Thus, we prove the coincidence between the variation of the $D$-dimensional charged black hole and the first law of thermodynamics under the charged particle absorption.

The second law of thermodynamics predicts the increase of entropy of the black hole for an irreversible process. As the charged particle absorption is an irreversible process, the entropy becomes greater than that before the absorption. The validity of this statement is easily checked by the sign of $d S_{\mathrm{h}}$ in eq. (3.16). Moreover, we obtain the violation of the second law of thermodynamics in parameter ranges. Specifically, the denominator of $d S_{\mathrm{h}}$ has a negative value for the extremal black hole. So,

$$
r_{\mathrm{h}}^{D+2}-2 M r_{\mathrm{h}}^{3} \ell^{2}+2 r_{\mathrm{h}}^{D} \ell^{2}=-\frac{(D-1) r_{\mathrm{h}}^{D+2}}{(D-3)}<0,
$$

which means that the entropy of the black hole decreases at least for the extremal case for all dimensions, $D \geq 4$. Therefore, the second law of thermodynamics is violated under the consideration of the $P V_{\mathrm{b}}$ term for the charged particle absorption. This behavior is interesting and observable only with the $P V_{\mathrm{b}}$ term in the first law of thermodynamics. Note that, without the $P V$ term, the second law of thermodynamics is always ensured when the first law of thermodynamics is satisfied [31]. Using $d S_{\mathrm{h}}$ in eq. (3.16), we investigate parameter ranges within which this violation occurs. The area of the violation depends on the spacetime dimensions. The parameter space $(Q, M)$ is limited because of the extremal condition for $D$-dimensional black holes in figure 1. As already shown in eq. (3.19), the decrease of entropy appears in ranges close to those of extremal black holes. In figure 1 (a), (b), and (c), we show parameter spaces of higher-dimensional black holes from four to six dimensions. These diagrams are similar, showing that the violation still exists in any dimension. This also implies that the entropy needs a correction term to resolve the violation when we consider thermodynamic pressure and volume. 


\section{Weak cosmic censorship conjecture with pressure and volume}

In consideration of thermodynamic volume, the charged particle absorption can reproduce the first law of thermodynamics in terms of enthalpy. However, although particle absorption is an irreversible process, we find the violation of the second law of thermodynamics in the process, and the entropy of the black hole decreases for extremal and near-extremal black holes. Without the $P V_{\mathrm{b}}$ term, the second law of thermodynamics is satisfied to validate the weak cosmic censorship conjecture under the absorption. Thus, owing to the violation of the second law with the $P V_{\mathrm{b}}$ term, we can expect that the cosmic censorship is affected by this term [32].

As the violation of the second law of thermodynamics occurs in extremal and nearextremal black holes, the changes in extremal and near-extremal black holes are very different from those in non-extremal ones. This change can be estimated from a behavior of the function $f(r) \equiv f\left(M_{\mathrm{b}}, Q_{\mathrm{b}}, \ell, r\right)$ in the metric of eq. (2.3). The function $f\left(M_{\mathrm{b}}, Q_{\mathrm{b}}, \ell, r\right)$ of the black hole has only one minimum point $r_{\min }$, which satisfies

$$
\left.f\left(M_{\mathrm{b}}, Q_{\mathrm{b}}, \ell, r\right)\right|_{r=r_{\min }} \equiv f_{\min }=\delta \leq 0,\left.\quad \partial_{r} f\left(M_{\mathrm{b}}, Q_{\mathrm{b}}, \ell, r\right)\right|_{r=r_{\min }} \equiv f_{\min }^{\prime}=0,
$$

with

$$
\left.\left(\partial_{r}\right)^{2} f\left(M_{\mathrm{b}}, Q_{\mathrm{b}}, \ell, r\right)\right|_{r=r_{\min }}>0 .
$$

The minimum value of the function $f\left(M_{\mathrm{b}}, Q_{\mathrm{b}}, \ell, r\right)$ is $\delta$, and $\delta=0$ for an extremal black hole. In addition, the inner and outer horizons are located around the minimum point. We explicitly denote variables $\left(M_{\mathrm{b}}, Q_{\mathrm{b}}, \ell\right)$ changed by the absorption. The conserved quantities of the particle infinitesimally change variables into $\left(M_{\mathrm{b}}+d M_{\mathrm{b}}, Q_{\mathrm{b}}+d Q_{\mathrm{b}}, \ell+d \ell\right)$. Then, owing to these changes, the locations of the minimum point and outer horizon are both infinitesimally shifted to $r_{\min } \rightarrow r_{\min }+d r_{\min }$ and $r_{h} \rightarrow r_{h}+d r_{h}$, respectively. Under these changes, the configuration of the black hole after the absorption can be expected from a change of the minimum value of the function $d f_{\min }$. Then, the moved minimum point satisfies

$$
\left.\partial_{r} f\left(M_{\mathrm{b}}+d M_{\mathrm{b}}, Q_{\mathrm{b}}+d Q_{b}, \ell+d \ell, r\right)\right|_{r=r_{\min }+d r_{\min }}=f_{\text {min }}^{\prime}+d f_{\text {min }}^{\prime}=0,
$$

which is in terms of variables with partial derivatives

$$
d f_{\min }^{\prime}=\frac{\partial f_{\min }^{\prime}}{\partial M_{\mathrm{b}}} d M_{\mathrm{b}}+\frac{\partial f_{\min }^{\prime}}{\partial Q_{\mathrm{b}}} d Q_{\mathrm{b}}+\frac{\partial f_{\min }^{\prime}}{\partial \ell} d \ell+\frac{\partial f_{\min }^{\prime}}{\partial r_{\min }} d r_{\min }=0,
$$

with

$$
\frac{\partial f_{\min }^{\prime}}{\partial M_{\mathrm{b}}}=\frac{16(D-3) \pi}{(D-2) \Omega_{D-2} r_{\min }^{D-2}}, \quad \frac{\partial f_{\min }^{\prime}}{\partial Q_{\mathrm{b}}}=-\frac{16(2 D-6) \pi Q}{(D-2) \Omega_{D-2} r_{\min }^{2 D-5}}, \quad \frac{\partial f_{\min }^{\prime}}{\partial \ell}=-\frac{4 r_{\min }}{\ell^{3}} .
$$

The value of the minimum at $r_{\min }+d r_{\min }$ becomes

$$
\begin{aligned}
\left.f\left(M_{b}+d M_{b}, Q_{b}+d Q_{b}, \ell+d \ell, r\right)\right|_{r=r_{\min }+d r_{\min }} & =f_{\min }+d f_{\min } \\
& =\delta+\left(\frac{\partial f_{\min }}{\partial M_{b}} d M_{b}+\frac{\partial f_{\min }}{\partial Q_{b}} d Q_{b}+\frac{\partial f_{\min }}{\partial \ell} d \ell\right),
\end{aligned}
$$




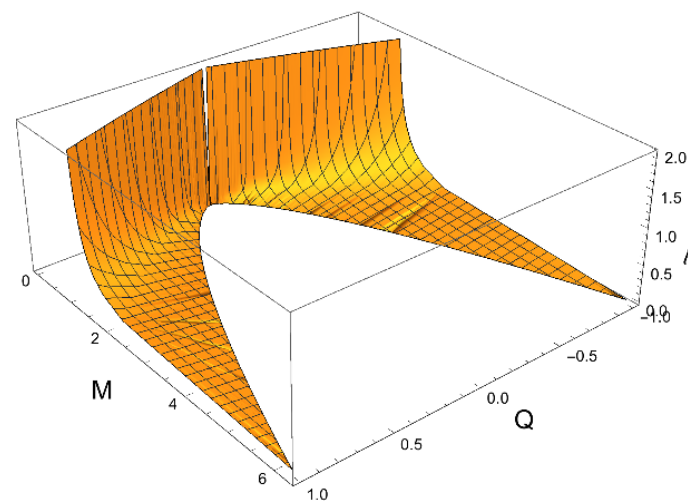

(a) $(Q, M, \ell)$ surface satisfying $\delta=0$.

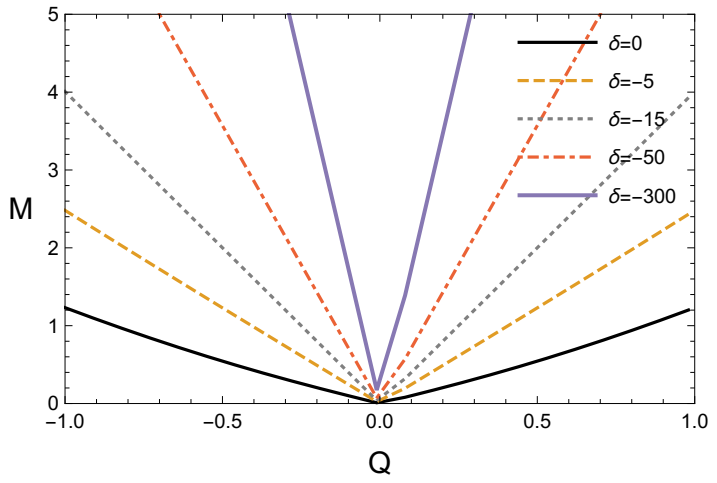

(b) $Q-M$ lines in the $\ell=1$ slice for values of $\delta$.

Figure 2. $(Q, M, \ell)$ surface and $Q-M$ diagram.

where we use $f_{\min }^{\prime}=0$ in eq. (4.1). Then, we can obtain $\left(d M_{\mathrm{b}}, d \ell\right)$ in terms of the particle charges $\left(q,\left|p^{r}\right|\right)$ under particle absorption. Owing to the location of the absorption, the outer horizon, the value of the minimum is obtained under $\left(r_{\min }, r_{\mathrm{h}}\right)$. However, this is too complex to analyze and express. Instead, we can impose the following condition for the near-extremal black hole

$$
\delta \rightarrow \delta_{\epsilon}, \quad r_{\mathrm{h}} \rightarrow r_{\min }+\epsilon .
$$

The outer horizon of the near-extremal black hole is located slightly to the right of the minimum point, and the minimum value is a very small negative value. This is given as $\left|\delta_{\epsilon}\right|, \epsilon \ll 1$. For the near-extremal black hole, the shifted minimum value is

$$
f_{\min }+d f_{\min }=\left(\delta_{\epsilon}+\frac{32 \pi r_{\min }^{5}\left(-1-(D-2) r_{\min }^{1-2 D}\left(-Q^{2} r_{\min }^{3}+M r_{\min }^{D}\right) \ell^{2}\right)\left|p^{r}\right|}{\Omega_{D-2}(D-3)(D-2)\left(r_{\min }^{D+2}-2 M r_{\min }^{3} \ell^{2}+2 r_{\min }^{D} \ell^{2}\right)}\right)+\mathcal{O}(\epsilon),
$$

where we have skipped the first order of $\epsilon$. To simplify this expansion, if we remove the $Q^{2}$ term by using $f_{\min }^{\prime}=0$, the expression becomes

$$
f_{\min }+d f_{\min }=\delta_{\epsilon}+\mathcal{O}\left(\epsilon^{2}\right)
$$

The first order of $\epsilon$ is also removed, and the minimum value of the extremal black hole is

$$
f_{\min }+d f_{\min }=0, \quad \delta_{\epsilon}=0, \quad \epsilon=0 .
$$

Therefore, extremal and near-extremal black holes stay at their minimum, because of which their phases cannot be changed, even if they are charged or discharged by the charged particle absorption. This result is quite different from that in cases without the $P V_{\mathrm{b}}$ term, where an extremal black hole is easily broken down into a non-extremal one by the absorption. Because the minimum value of the extremal black hole does not change, the varied extremal black hole can stay on the $\delta=0$ surface, as shown in figure 2 (a). Further, from eq. (3.19), to decrease the entropy, the extremal black hole should be contracted under the particle absorption in figure $2(\mathrm{~b})$. On the plane of $(Q, M, \ell)$, the phase of the extremal 
black hole moves three-dimensionally because the AdS radius is changed by the absorption. However, as shown in the $M-Q$ diagram of figure $2(\mathrm{~b})$, the extremal black hole stays on the extremal line. The value of $\delta$ of the near-extremal black hole is also invariant under the absorption.

\section{Summary}

We have investigated variations in the charged AdS black hole under charged particle absorption by considering the pressure as a cosmological constant. It is known that the thermal conjugate of pressure is the volume of the black hole inside of the horizon. However, because the cosmological constant is not a variable in the action and equations of motion, the dynamical effect is not easy to predict with the pressure term. To elucidate the effect of the pressure and volume terms, we consider an infinitesimal variation in the black hole by a charged particle. This is probably the only way to demonstrate the effect of an external particle without using the equations of motion. Then, when the particle is absorbed into the black hole, the conserved quantities of the black hole vary by as much as the conserved quantities of the charged particle. The change in the black hole exactly corresponds to the first law of thermodynamics in terms of enthalpy. However, the second law of thermodynamics is violated for extremal and near-extremal black holes, in which the entropy decreases under the absorption. This implies that, at least, the entropy of the black hole needs a correction, which should not be proportional to the outer horizon. The violation of the second law of thermodynamics can be related to the weak cosmic censorship conjecture, which is related to the stability of the horizon. The stability can be shown from the change of the minimum value of the function $f(M, Q, \ell, r)$ under the absorption. Interestingly, the variation of the minimum value is quite different from that in the case without the pressure term. The minimum value of the function $f(M, Q, \ell, r)$ is not changed for extremal and near-extremal black holes under the absorption. This implies that extremal or nearextremal black holes stay as they are after the absorption of an external particle. Thus, even if extremal or near-extremal black holes are charged or discharged by the absorption, they maintain their extremality or near-extremality. This result cannot be seen in charged particle absorption without the thermodynamic pressure and volume term. In addition, owing to the maintenance of its minimum value, the extremal black hole cannot be overcharged in the process. This ensures the stability of the horizon under the charged particle absorption.

\section{Acknowledgments}

This work was supported by Basic Science Research Program through the National Research Foundation of Korea (NRF) funded by the Ministry of Science, ICT \& Future Planning (NRF-2015R1C1A1A02037523).

Open Access. This article is distributed under the terms of the Creative Commons Attribution License (CC-BY 4.0), which permits any use, distribution and reproduction in any medium, provided the original author(s) and source are credited. 


\section{References}

[1] S.W. Hawking, Particle creation by black holes, Commun. Math. Phys. 43 (1975) 199 [Erratum ibid. 46 (1976) 206] [INSPIRE].

[2] S.W. Hawking, Black holes and thermodynamics, Phys. Rev. D 13 (1976) 191 [INSPIRE].

[3] D. Christodoulou, Reversible and irreversible transformations in black hole physics, Phys. Rev. Lett. 25 (1970) 1596 [INSPIRE].

[4] D. Christodoulou and R. Ruffini, Reversible transformations of a charged black hole, Phys. Rev. D 4 (1971) 3552 [INSPIRE].

[5] L. Smarr, Mass formula for Kerr black holes, Phys. Rev. Lett. 30 (1973) 71 [Erratum ibid. 30 (1973) 521] [INSPIRE].

[6] J.M. Bardeen, Kerr metric black holes, Nature 226 (1970) 64 [InSPIRE].

[7] J.D. Bekenstein, Black holes and entropy, Phys. Rev. D 7 (1973) 2333 [InSPIRE].

[8] J.D. Bekenstein, Generalized second law of thermodynamics in black hole physics, Phys. Rev. D 9 (1974) 3292 [INSPIRE].

[9] R. Penrose, Gravitational collapse and space-time singularities, Phys. Rev. Lett. 14 (1965) 57 [INSPIRE].

[10] R. Penrose, Gravitational collapse: the role of general relativity, Riv. Nuovo Cim. 1 (1969) 252 [Gen. Rel. Grav. 34 (2002) 1141] [INSPIRE].

[11] R. Wald, Gedanken experiments to destroy a black hole, Annals Phys. 82 (1974) 548.

[12] T. Jacobson and T.P. Sotiriou, Over-spinning a black hole with a test body, Phys. Rev. Lett. 103 (2009) 141101 [Erratum ibid. 103 (2009) 209903] [arXiv:0907.4146] [INSPIRE].

[13] E. Barausse, V. Cardoso and G. Khanna, Test bodies and naked singularities: is the self-force the cosmic censor?, Phys. Rev. Lett. 105 (2010) 261102 [arXiv:1008.5159] [INSPIRE].

[14] E. Barausse, V. Cardoso and G. Khanna, Testing the cosmic censorship conjecture with point particles: the effect of radiation reaction and the self-force, Phys. Rev. D 84 (2011) 104006 [arXiv:1106.1692] [INSPIRE].

[15] M. Colleoni, L. Barack, A.G. Shah and M. van de Meent, Self-force as a cosmic censor in the Kerr overspinning problem, Phys. Rev. D 92 (2015) 084044 [arXiv:1508.04031] [INSPIRE].

[16] V.E. Hubeny, Overcharging a black hole and cosmic censorship, Phys. Rev. D 59 (1999) 064013 [gr-qc/9808043] [INSPIRE].

[17] S. Isoyama, N. Sago and T. Tanaka, Cosmic censorship in overcharging a Reissner-Nordström black hole via charged particle absorption, Phys. Rev. D 84 (2011) 124024 [arXiv:1108.6207] [INSPIRE].

[18] M. Colleoni and L. Barack, Overspinning a Kerr black hole: the effect of self-force, Phys. Rev. D 91 (2015) 104024 [arXiv:1501.07330] [INSPIRE].

[19] M. Bouhmadi-Lopez, V. Cardoso, A. Nerozzi and J.V. Rocha, Black holes die hard: can one spin-up a black hole past extremality?, Phys. Rev. D 81 (2010) 084051 [arXiv:1003.4295] [INSPIRE].

[20] S. Hod, A note on black-hole physics, cosmic censorship and the charge-mass relation of atomic nuclei, Class. Quant. Grav. 33 (2016) 037001 [INSPIRE]. 
[21] J. Natario, L. Queimada and R. Vicente, Test fields cannot destroy extremal black holes, Class. Quant. Grav. 33 (2016) 175002 [arXiv:1601.06809] [INSPIRE].

$[22]$ G.T. Horowitz, J.E. Santos and B. Way, Evidence for an electrifying violation of cosmic censorship, Class. Quant. Grav. 33 (2016) 195007 [arXiv: 1604.06465] [INSPIRE].

[23] K. Düztaş, Overspinning BTZ black holes with test particles and fields, Phys. Rev. D 94 (2016) 124031 [arXiv:1701.07241] [INSPIRE].

[24] J. Sorce and R.M. Wald, Gedanken experiments to destroy a black hole II: Kerr-Newman black holes cannot be over-charged or over-spun, Phys. Rev. D 96 (2017) 104014 [arXiv: 1707.05862] [INSPIRE].

[25] S. Gao and Y. Zhang, Destroying extremal Kerr-Newman black holes with test particles, Phys. Rev. D 87 (2013) 044028 [arXiv:1211.2631] [InSPIRE].

[26] J.V. Rocha, R. Santarelli and T. Delsate, Collapsing rotating shells in Myers-Perry-AdS 5 spacetime: a perturbative approach, Phys. Rev. D 89 (2014) 104006 [arXiv:1402.4161] [INSPIRE].

[27] J.V. Rocha and R. Santarelli, Flowing along the edge: spinning up black holes in AdS spacetimes with test particles, Phys. Rev. D 89 (2014) 064065 [arXiv:1402.4840] [INSPIRE].

[28] B. McInnes and Y.C. Ong, A note on physical mass and the thermodynamics of AdS-Kerr black holes, JCAP 11 (2015) 004 [arXiv: 1506.01248] [INSPIRE].

[29] J.V. Rocha and V. Cardoso, Gravitational perturbation of the BTZ black hole induced by test particles and weak cosmic censorship in AdS spacetime, Phys. Rev. D 83 (2011) 104037 [arXiv:1102.4352] [INSPIRE].

[30] B. Gwak, Stability of horizon in warped AdS black hole via particle absorption, arXiv: 1707.09128 [INSPIRE].

[31] B. Gwak and B.-H. Lee, Cosmic censorship of rotating anti-de Sitter black hole, JCAP 02 (2016) 015 [arXiv: 1509.06691] [INSPIRE].

[32] B. Gwak, Cosmic censorship conjecture in Kerr-Sen black hole, Phys. Rev. D 95 (2017) 124050 [arXiv: 1611.09640] [INSPIRE].

[33] J.M. Maldacena, The large- $N$ limit of superconformal field theories and supergravity, Int. J. Theor. Phys. 38 (1999) 1113 [hep-th/9711200] [INSPIRE].

[34] S.S. Gubser, I.R. Klebanov and A.M. Polyakov, Gauge theory correlators from noncritical string theory, Phys. Lett. B 428 (1998) 105 [hep-th/9802109] [InSPIRE].

[35] E. Witten, Anti-de Sitter space and holography, Adv. Theor. Math. Phys. 2 (1998) 253 [hep-th/9802150] [INSPIRE].

[36] O. Aharony, S.S. Gubser, J.M. Maldacena, H. Ooguri and Y. Oz, Large-N field theories, string theory and gravity, Phys. Rept. 323 (2000) 183 [hep-th/9905111] [INSPIRE].

[37] E. Witten, Anti-de Sitter space, thermal phase transition and confinement in gauge theories, Adv. Theor. Math. Phys. 2 (1998) 505 [hep-th/9803131] [inSPIRE].

[38] J. Babington, J. Erdmenger, N.J. Evans, Z. Guralnik and I. Kirsch, Chiral symmetry breaking and pions in nonsupersymmetric gauge/gravity duals, Phys. Rev. D 69 (2004) 066007 [hep-th/0306018] [INSPIRE].

[39] M. Kruczenski, D. Mateos, R.C. Myers and D.J. Winters, Towards a holographic dual of large- $N_{c} Q C D$, JHEP 05 (2004) 041 [hep-th/0311270] [INSPIRE]. 
[40] T. Sakai and S. Sugimoto, More on a holographic dual of QCD, Prog. Theor. Phys. 114 (2005) 1083 [hep-th/0507073] [inSPIRE].

[41] J. Erlich, E. Katz, D.T. Son and M.A. Stephanov, QCD and a holographic model of hadrons, Phys. Rev. Lett. 95 (2005) 261602 [hep-ph/0501128] [INSPIRE].

[42] S.A. Hartnoll, C.P. Herzog and G.T. Horowitz, Building a holographic superconductor, Phys. Rev. Lett. 101 (2008) 031601 [arXiv:0803.3295] [INSPIRE].

[43] S.A. Hartnoll, C.P. Herzog and G.T. Horowitz, Holographic superconductors, JHEP 12 (2008) 015 [arXiv:0810.1563] [INSPIRE].

[44] K. Jensen, Chiral anomalies and AdS/CMT in two dimensions, JHEP 01 (2011) 109 [arXiv: 1012.4831] [INSPIRE].

[45] T. Andrade, J.I. Jottar and R.G. Leigh, Boundary conditions and unitarity: the Maxwell-Chern-Simons system in $A d S_{3} / C F T_{2}$, JHEP 05 (2012) 071 [arXiv:1111.5054] [INSPIRE].

[46] H.-C. Chang, M. Fujita and M. Kaminski, From Maxwell-Chern-Simons theory in AdS 3 towards hydrodynamics in $1+1$ dimensions, JHEP 10 (2014) 118 [arXiv:1403.5263] [INSPIRE].

[47] L.-Y. Hung and A. Sinha, Holographic quantum liquids in $1+1$ dimensions, JHEP 01 (2010) 114 [arXiv:0909.3526] [INSPIRE].

[48] R.A. Davison, M. Goykhman and A. Parnachev, AdS/CFT and Landau Fermi liquids, JHEP 07 (2014) 109 [arXiv: 1312.0463] [inSPIRE].

[49] C. Teitelboim, The cosmological constant as a thermodynamic black hole parameter, Phys. Lett. B 158 (1985) 293 [INSPIRE].

[50] J.D. Brown and C. Teitelboim, Neutralization of the cosmological constant by membrane creation, Nucl. Phys. B 297 (1988) 787 [INSPIRE].

[51] M.M. Caldarelli, G. Cognola and D. Klemm, Thermodynamics of Kerr-Newman-AdS black holes and conformal field theories, Class. Quant. Grav. 17 (2000) 399 [hep-th/9908022] [INSPIRE].

[52] T. Padmanabhan, Classical and quantum thermodynamics of horizons in spherically symmetric space-times, Class. Quant. Grav. 19 (2002) 5387 [gr-qc/0204019] [INSPIRE].

[53] B.P. Dolan, The cosmological constant and the black hole equation of state, Class. Quant. Grav. 28 (2011) 125020 [arXiv: 1008.5023] [INSPIRE].

[54] M. Cvetič, G.W. Gibbons, D. Kubiznak and C.N. Pope, Black hole enthalpy and an entropy inequality for the thermodynamic volume, Phys. Rev. D 84 (2011) 024037 [arXiv: 1012.2888] [INSPIRE].

[55] D. Kastor, S. Ray and J. Traschen, Enthalpy and the mechanics of AdS black holes, Class. Quant. Grav. 26 (2009) 195011 [arXiv:0904.2765] [INSPIRE].

[56] B.P. Dolan, Pressure and volume in the first law of black hole thermodynamics, Class. Quant. Grav. 28 (2011) 235017 [arXiv:1106.6260] [INSPIRE].

[57] D. Kubiznak and R.B. Mann, P-V criticality of charged AdS black holes, JHEP 07 (2012) 033 [arXiv: 1205.0559 ] [INSPIRE].

[58] C.V. Johnson, Holographic heat engines, Class. Quant. Grav. 31 (2014) 205002 [arXiv: 1404.5982] [INSPIRE]. 
[59] E. Caceres, P.H. Nguyen and J.F. Pedraza, Holographic entanglement entropy and the extended phase structure of STU black holes, JHEP 09 (2015) 184 [arXiv:1507.06069] [INSPIRE].

[60] A. Karch and B. Robinson, Holographic black hole chemistry, JHEP 12 (2015) 073 [arXiv: 1510.02472] [INSPIRE].

[61] D. Kubiznak, R.B. Mann and M. Teo, Black hole chemistry: thermodynamics with Lambda, Class. Quant. Grav. 34 (2017) 063001 [arXiv:1608.06147] [INSPIRE].

[62] R.A. Hennigar, F. McCarthy, A. Ballon and R.B. Mann, Holographic heat engines: general considerations and rotating black holes, Class. Quant. Grav. 34 (2017) 175005 [arXiv: 1704.02314] [INSPIRE].

[63] S.-W. Wei and Y.-X. Liu, Charged AdS black hole heat engines, arXiv:1708.08176 [INSPIRE].

[64] R. Gregory, D. Kastor and J. Traschen, Black hole thermodynamics with dynamical lambda, JHEP 10 (2017) 118 [arXiv: 1707.06586] [INSPIRE].

[65] B. Gwak, Collision of two rotating Hayward black holes, Eur. Phys. J. C 77 (2017) 482 [arXiv: 1703.10154] [INSPIRE].

[66] Y. Tian and X.-N. Wu, Dynamics of gravity as thermodynamics on the spherical holographic screen, Phys. Rev. D 83 (2011) 021501 [arXiv:1007.4331] [INSPIRE].

[67] B.P. Dolan, Where is the PdV term in the fist law of black hole thermodynamics?, arXiv:1209.1272 [INSPIRE].

[68] B.P. Dolan, D. Kastor, D. Kubiznak, R.B. Mann and J. Traschen, Thermodynamic volumes and isoperimetric inequalities for de Sitter black holes, Phys. Rev. D 87 (2013) 104017 [arXiv: 1301.5926] [INSPIRE].

[69] B. Carter, Hamilton-Jacobi and Schrödinger separable solutions of Einstein's equations, Commun. Math. Phys. 10 (1968) 280 [INSPIRE]. 